\title{
Magnetic resonance imaging of the axial structures of the brain
}

Two of the author's answers in the online version of this Endgames Anatomy Quiz (BMJ 2012;345:e5268, doi:10.1136/ bmj.e5268) by Raymond Chung were incorrect. Answer D

should have been the putamen (not the "Globus pallidus-left") and answer E should have been the posterior limb of the internal capsule (not the "Putamen-left").

Cite this as: BMJ 2012;345:e5848

๑ BMJ Publishing Group Ltd 2012 